\title{
An Extension Theorem for Terraces
}

\author{
M. A. Ollis* and Devin T. Willmott \\ Marlboro College, P.O. Box A, Marlboro \\ Vermont 05344, USA
}

Submitted: Mar 23, 2012; Accepted: May 10, 2013; Published: May 24, 2013

\begin{abstract}
We generalise an extension theorem for terraces for abelian groups to apply to nonabelian groups with a central subgroup isomorphic to the Klein 4-group $V$. We also give terraces for three of the non-abelian groups of order a multiple of 8 that have a cyclic subgroup of index 2 that may be used in the extension theorem. These results imply the existence of terraces for many groups that were not previously known to be terraced, including 27 non-abelian groups of order 64 and all groups of the form $V^{s} \times D_{8 t}$ for all $s$ and all $t>1$ where $D_{8 t}$ is the dihedral group of order $8 t$.
\end{abstract}

AMS 2010 Subject Classification. Primary: 20D60. Secondary: 05B99.

Keywords: 2-sequencing, Bailey's conjecture, extendable terrace, rotational terrace, terrace.

\section{Introduction}

Let $G$ be a group of order $n$ and let $\mathbf{a}=\left(a_{1}, a_{2}, \ldots, a_{n}\right)$ be an arrangement of all of the elements of $G$. Define $\mathbf{b}=\left(b_{1}, b_{2}, \ldots, b_{n-1}\right)$ by $b_{i}=a_{i}^{-1} a_{i+1}$. If each involution of $G$ appears once in $\mathbf{b}$ and there are two appearances from each set $\left\{g, g^{-1}: g^{2} \neq e\right\}$ in $\mathbf{b}$ then $\mathbf{a}$ is a terrace for $G$ and $\mathbf{b}$ is its associated 2-sequencing. If a group has a terrace then it is terraced. Left-multiplying each element of a terrace by any element of the group produces another terrace for the group; choosing $a_{1}^{-1}$ gives a terrace with the identity, $e$, as the first element-such a terrace is called basic.

Terraces for cyclic groups were implicitly used by Williams in [13] and the concept was formally defined and extended to arbitrary groups by Bailey [3]. They were originally of interest because the Cayley table of a group may be presented as a quasi-complete Latin square if and only if the group is terraced [3] but have since been used for other applications and studied as objects of interest in their own right. The purpose of this paper is to move closer to a proof of Bailey's Conjecture:

\footnotetext{
${ }^{*}$ Corresponding author, email address: matt@marlboro.edu .
} 
Conjecture 1 [3] All groups, except the non-cyclic elementary abelian 2-groups, are terraced.

It is known that the non-cyclic elementary abelian 2-groups are not terraced [3].

Example 1 Let $\mathbb{Z}_{n}$ be the additively-written cyclic group of order $n$. The Lucas-WaleckiWilliams terrace (so-called because it was implicitly used by Lucas and Walecki [7] for even $n$ and by Williams [13] for all $n)$ for $\mathbb{Z}_{n}$ is $(0,1, n-1,2, n-2, \ldots)$ and has associated 2sequencing $(1, n-2,3, n-4, \ldots)$.

There have been two main lines of attack on Bailey's Conjecture. First, one may directly construct terraces for a particular family of groups. Second, one can produce theorems that build a terrace for a group out of terraces for smaller groups. The most powerful example of the second approach is the following result:

Theorem 1 [1,2] Let $G$ be a group with normal subgroup $N$. If $N$ has odd index and is terraced, then $G$ is terraced. If $N$ has odd order and $G / N$ is terraced then $G$ is terraced.

In [9] a theorem that constructed a terrace for an abelian group $G$ that has a subgroup of order 4 and a particular type of terrace for the quotient group was presented. This theorem is not fully correct in the case when the subgroup of order 4 is cyclic; see [11] in which the error is corrected and it is shown that all of the groups claimed to be terraced are indeed terraced. In the next section we present a more general version of the correct case (when the subgroup is isomorphic to $V$, the Klein 4-group) that applies to many non-abelian groups.

The extension result in the next section allows us to find terraces for a considerable array of previously unterraced groups. As input the theorem requires terraces with particular properties; some such terraces are catalogued in Section 3.

\section{The extension theorem}

We first define the properties we require of a terrace to be used in the theorem. Let $K$ be a group of order $m \geqslant 6$ and let $\mathbf{a}=\left(a_{1}, a_{2}, \ldots, a_{m}\right)$ be a basic terrace for $K$. If $a_{m}=a_{2}^{2}$ and $a_{j-1} a_{j+1}=a_{j}=a_{j+1} a_{j-1}$ for some $5 \leqslant j<m$ then $\mathbf{a}$ is extendable.

An important intermediary object is an R-terrace, or rotational terrace. Following the convention of earlier papers we write circular lists in square brackets and consider the subscripts to be calculated modulo the length of the list. Let $K$ be a group of order $m$ and let $\mathbf{a}=\left[a_{1}, a_{2}, \ldots, a_{m-1}\right]$ be a circular arrangement of the non-identity elements of $K$. Define $\mathbf{b}=\left[b_{1}, b_{2}, \ldots, b_{m-1}\right]$ by $b_{i}=a_{i}^{-1} a_{i+1}$ for $1 \leqslant i \leqslant m-1$. If $\mathbf{b}$ contains exactly one occurrence of each involution of $K$ and exactly two occurrences from each set $\left\{k, k^{-1}: k^{2} \neq e\right\}$ then a is a rotational terrace or $R$-terrace for $K$ and $\mathbf{b}$ is the associated rotational 2-sequencing or $R$-2-sequencing of $K$. If there are no repeats among the elements of $\mathbf{b}$ then the R-terrace is directed and the R-2-sequencing is an $R$-sequencing. Further, if $a_{1}=a_{m-1} a_{2}=a_{2} a_{m-1}$ then a is a standard $R^{*}$-terrace for $K$ and if $b_{r}=a_{r+1}^{-1}$ for some $r$ then $r$ is a right match-point 
of $\mathbf{b}$. Note that a standard $\mathrm{R}^{*}$-terrace cannot have an $\mathrm{R}$-2-sequencing with 1 as a right match-point.

Standard $\mathrm{R}^{*}$-terraces whose $\mathrm{R}$-2-sequencings have particular right match-points and extendable terraces are equivalent: The circular list $\left[a_{1}, a_{2}, \ldots, a_{m-1}\right]$ is a standard $\mathrm{R}^{*}$-terrace whose R-2-sequencing has a right match-point $r$ for some $2 \leqslant r \leqslant m-3$ if and only if

$$
\left(e, a_{r+1}, a_{r+2}, \ldots, a_{m-1}, a_{1}, a_{2}, \ldots, a_{r}\right)
$$

is an extendable terrace.

The following lemma restricts which groups may have an extendable terrace.

Lemma 1 [9] If the order of $G$ is congruent to 2 modulo 4 then $G$ does not have a rotational terrace.

We can now prove our main result. The Klein 4-group is the non-cyclic group of order 4 and a subgroup is central if each of its elements commutes with every element of the group (that is, it is contained in the centre of the group).

Theorem 2 Let $G$ be a group with a central subgroup $V$ of index $m \geqslant 7$, where $V$ is isomorphic to the Klein 4-group. Suppose $G / V$ has a standard $R^{*}$-terrace $\left[K_{1}, K_{2}, \ldots, K_{m-1}\right]$ whose $R$-2-sequencing has a match-point $r$ for some $2 \leqslant r \leqslant m-3$ and such that there is a pair of elements, one in $K_{2}$ and one in $K_{m-1}$, that commute. Then $G$ has an extendable terrace.

Proof. Choose coset representatives $k_{i}$, for $1 \leqslant i \leqslant m-1$, such that $k_{i} \in K_{i}$ and that both $k_{m-1} k_{2}=k_{1}=k_{2} k_{m-1}$ and $k_{r}^{-1} k_{r+1}=k_{r+1}^{-1}$. These two criteria potentially interact if $r=2$. In this case, choose any $k_{3} \in K_{3}$, set $k_{2}=k_{3}^{2}$ and then there is a $k_{m-1} \in K_{m-1}$ that commutes with $k_{2}$ : if $\ell_{2} \in K_{2}$ and $\ell_{m-1} \in K_{m-1}$ are the commuting elements we know to exist then there is a $v_{0} \in V$ with $k_{2}=v_{0} \ell_{2}$ and this commutes with $\ell_{m-1}$, which we may set to be $k_{m-1}$, by the centrality of $V$.

Note that each element of $G$ is uniquely expressible in the form $v k$ for $v \in V$ and $k \in\left\{e, k_{1}, k_{2}, \ldots, k_{m-1}\right\}$.

We build the standard $\mathrm{R}^{*}$-terrace by showing the lists of the $v$ components and $k$ components separately. Let $V=\left\{e, v_{1}, v_{2}, v_{3}\right\}$, then $\left[v_{1}, v_{2}, v_{3}\right]$ is an R-terrace for $V$ (that is, any circular list of the non-identity elements of $V$ is an R-terrace). We list the $v$ components as the rows of a $4 \times m$ matrix. Let $\left(v_{1}, v_{2}, v_{3}\right)_{t-1}$ denote $t-1$ repetitions of the sequence $\left(v_{1}, v_{2}, v_{3}\right)$, and similarly for other subscripted sequences. There are three slightly different matrices for the $v$ components as $m$ varies modulo 3 .

Case 1: $m=3 t$ for $t \geqslant 3$. Take

$$
\left[\begin{array}{ccccccc}
e & e & \cdots & & e & v_{2} & v_{1} \\
v_{3} & \left(v_{1}, v_{2}, v_{3}\right)_{t-2} & v_{1} & v_{3} & v_{3} & v_{2} & v_{1} \\
v_{3} & v_{2} & \left(v_{3}, v_{1}, v_{2}\right)_{t-2} & v_{3} & v_{2} & v_{1} & v_{3} \\
v_{2} & v_{1} & \left(v_{2}, v_{3}, v_{1}\right)_{t-2} & v_{2} & v_{1} & e &
\end{array}\right]
$$


to be the $v$ component matrix.

Case 2: $m=3 t+1$ for $t \geqslant 2$. Take

$$
\left[\begin{array}{ccccc}
e & e & \cdots & v_{2} & v_{1} \\
v_{3} & \left(v_{2}, v_{1}, v_{3}\right)_{t-1} & v_{3} & v_{2} & v_{1} \\
v_{3} & v_{2} & \left(v_{1}, v_{3}, v_{2}\right)_{t-1} & v_{1} & v_{3} \\
v_{2} & v_{1} & \left(v_{3}, v_{2}, v_{1}\right)_{t-1} & e &
\end{array}\right]
$$

to be the $v$ component matrix.

Case 3: $m=3 t+2$ for $t \geqslant 2$. Take

$$
\left[\begin{array}{cccccc}
e & e & \cdots & e & v_{2} & v_{1} \\
v_{3} & \left(v_{2}, v_{1}, v_{3}\right)_{t-1} & v_{2} & v_{3} & v_{2} & v_{1} \\
v_{3} & v_{2} & \left(v_{1}, v_{3}, v_{2}\right)_{t-1} & v_{1} & v_{1} & v_{3} \\
v_{2} & v_{1} & \left(v_{3}, v_{2}, v_{1}\right)_{t-1} & v_{3} & e &
\end{array}\right]
$$

to be the $h$ component matrix.

For each of the above cases the $k$ component matrix is

$$
\left[\begin{array}{cccccc}
k_{1} & k_{2} & \ldots & & k_{m-1} & e \\
k_{2} & k_{3} & \ldots & k_{m-1} & k_{1} & k_{1} \\
k_{1} & k_{2} & \ldots & & k_{m-1} & e \\
e & k_{2} & k_{3} & \ldots & k_{m-1} &
\end{array}\right]
$$

As $V$ is central in $G$ and $k_{m-1} k_{2}=k_{1}=k_{2} k_{m-1}$ we get the following matrix of quotients in the $k$ component:

$$
\left[\begin{array}{ccccccc}
k_{1}^{-1} k_{2} & k_{2}^{-1} k_{3} & \ldots & \ldots & k_{m-2}^{-1} k_{m-1} & k_{1}^{-1} k_{2} & k_{m-1}^{-1} k_{1} \\
k_{2}^{-1} k_{3} & k_{3}^{-1} k_{4} & \ldots & k_{m-2}^{-1} k_{m-1} & k_{m-1}^{-1} k_{1} & e & e \\
k_{1}^{-1} k_{2} & k_{2}^{-1} k_{3} & \ldots & \ldots & k_{m-2}^{-1} k_{m-1} & k_{1}^{-1} k_{2} & e \\
k_{m-1}^{-1} k_{1} & k_{2}^{-1} k_{3} & k_{3}^{-1} k_{4} & \ldots & k_{m-2}^{-1} k_{m-1} & k_{m-1}^{-1} k_{1} &
\end{array}\right]
$$

Each repeated sequence in the $v$ component matrix is a directed R-terrace and so when the quotient matrices are combined we get a sequence that obeys the conditions of an R-2sequencing.

Further, as the first two entries and the last entry of every $v$ component matrix is $e$, it follows from our choices of $k_{1}, k_{2}$, and $k_{m-1}$ that the R-terrace is a standard $\mathrm{R}^{*}$-terrace. As the first $m-2$ entries of every $v$ component matrix are all $e$, our choices of $k_{r}$ and $k_{r+1}$ give us the match-point we require in position $r$ of the R-2-sequencing.

The awkward condition in Theorem 2 regarding commuting elements in commuting cosets is automatically satisfied in the cases where we have appropriate direct products or abelian (sub)groups. Hence an immediate consequence of the theorem is: 
Corollary 1 Let $A$ be an abelian 2-group that has a normal series with all factors isomorphic to the Klein 4-group and let $K$ be a group with an extendable terrace. Then $A \times K$ has an extendable terrace. In particular, $\mathbb{Z}_{2}^{2 s} \times K$ has an extendable terrace for all $s$.

Our goal now is to construct extendable terraces for as many groups as possible.

\section{$3 \quad$ Extendable terraces}

The following results for abelian groups are established in $[9,10,11]$ :

- The cyclic group $\mathbb{Z}_{n}$ has an extendable terrace if and only if $n \geqslant 7$ and $n$ is not twice an odd number.

- All abelian 2-groups of order at least 8, except the elementary abelian 2-groups, have an extendable terrace.

- Let $p$ be an odd prime. The group $\mathbb{Z}_{2}^{2 t} \times \mathbb{Z}_{p}$ has an extendable terrace unless $t=0$ and $p \leqslant 5$.

- The groups $\mathbb{Z}_{2}^{2 t+1} \times \mathbb{Z}_{3}$ and $\mathbb{Z}_{2}^{2 t+1} \times \mathbb{Z}_{5}$ have an extendable terrace for all $t \geqslant 1$.

Other than the unterraceable elementary abelian 2-groups, these results and Theorem 1 now give terraces for all abelian groups except for those of order coprime to 15 with elementary abelian Sylow 2-subgroup of order $2^{2 t+1}$ for $t \geqslant 1[9,10,11]$. When $t \geqslant 2$ it is known that these groups are terraced [10].

In this section we present extendable terraces for each of three non-abelian groups of order $8 t$ with $t \geqslant 2$ : the dihedral group $D_{8 t}$, the semidihedral group $S_{8 t}$ and a third group that also has a cyclic subgroup of index 2 but does not appear to have a common name in the literature - we denote it $M_{8 t}$ following Gorenstein's use, reported in [6], of the letter $M$ (but with a different subscript convention) for this group when it has order a power of 2 . For even $t$, other than finitely many small cases, the terraces given for $S_{8 t}$ and $M_{8 t}$ are the first known. Here are presentations for these groups:

$$
\begin{aligned}
D_{8 t} & =\left\langle u, v: u^{4 t}=e=v^{2}, v u=u^{4 t-1} v\right\rangle \\
S_{8 t} & =\left\langle u, v: u^{4 t}=e=v^{2}, v u=u^{2 t-1} v\right\rangle \\
M_{8 t} & =\left\langle u, v: u^{4 t}=e=v^{2}, v u=u^{2 t+1} v\right\rangle
\end{aligned}
$$

Before constructing the desired terraces we introduce a related concept and prove a lemma that is crucial to the construction.

An arrangement $\mathbf{g}=\left(g_{1}, g_{2}, \ldots, g_{n}\right)$ of the integers $\{0,1, \ldots, n-1\}$ is a graceful sequence of length $n$ if each element of the set $\{1,2, \ldots, n-1\}$ can be written $\left|g_{i+1}-g_{i}\right|$ for some $i$. This is equivalent to the notion of a graceful labelling of a path in graph theory [4]. If $\mathbf{g}$ is a graceful sequence then so are its reverse $\left(g_{n}, g_{n-1}, \ldots, g_{1}\right)$ and its complement $((n-1)-$ $\left.g_{1},(n-1)-g_{2}, \ldots,(n-1)-g_{n}\right)$. Considered to be a sequence in $\mathbb{Z}_{n}$ rather than $\mathbb{Z}$ a graceful sequence is a terrace, called a graceful terrace. 
Example 2 The negated LWW terrace for $\mathbb{Z}_{n}$, obtained by negating each element of the $L W W$ terrace of Example 1, is a graceful terrace.

Lemma 2 For all $t \geqslant 2$ there is a graceful sequence of length $2 t-1$ with endpoints $t-2$ and $2 t-3$.

Proof. When $t \equiv 5(\bmod 6)$ we use the complement of the "3-twizzler" graceful terrace described in [12]. The 3-twizzler terrace is obtained from the negated LWW terrace for $\mathbb{Z}_{2 t-1}$ by dividing the terrace into subsequences of length 3 and reversing ("twizzling") each of them. After taking the complement we have:

$$
\underbrace{2 t-3,0,2 t-2}, \underbrace{2,2 t-4,1}, \ldots, \underbrace{t-1, t, t-2} .
$$

When $t \equiv 2(\bmod 6)$ the complement of 3 -twizzler terrace begins the same way but ends $t-1, t-2, t$. Switching the last two elements preserves the gracefulness of the sequence and gives us the $t-2$ that we need as an endpoint.

When $t \equiv 0(\bmod 3)$ we can use the complement of the "imperfect 3 -twizzler" graceful terrace of [12]. In Preece's imperfect 3-twizzler terrace all but the final two elements are obtained by 3 -twizzling as above. Here is its complement:

$$
\underbrace{2 t-3,0,2 t-2}, \underbrace{2,2 t-4,1}, \ldots, \underbrace{t, t-3, t+1}, t-1, t-2 .
$$

Finally, when $t \equiv 1(\bmod 3)$ we give a new graceful terrace using similar ideas. We begin as in the previous cases by twizzling subsequences of length 3 from the negated LWW graceful terrace, however this time we stop with 7 elements remaining and rearrange those to give a final element of $t-2$ while preserving the gracefulness of the sequence:

$$
\underbrace{2 t-3,0,2 t-2}_{t+1, t-3, t+2, t-4, t-1, t, t-2 .}, \underbrace{2,2 t-4,1}, \ldots, \underbrace{t-5, t+3, t-6}_{t},
$$

This completes the proof.

Theorem 3 The groups $D_{8 t}, S_{8 t}$ and $M_{8 t}$ have an extendable terrace for all $t \geqslant 2$.

Proof. The similar structure of the three groups allows us to use a slightly unusual approach. We give a sequence of elements of the form $u^{x} v^{y}$ with $0 \leqslant x \leqslant 4 t-1$ and $y \in\{0,1\}$ and this sequence is a terrace regardless of to which group we interpret the elements belonging.

The terrace takes the form $\mathbf{a}=\left(e, \alpha, \beta, u^{2 t}, v, \gamma, u^{t} v, \delta\right)$, where each Greek letter represents a sequence of elements. With the exception of $\delta$, each of these sequences can be expressed in a "zigzag" pattern. The partial terrace up to $u^{t} v$ is given in Table 1. 
Table 1: Partial extendable terrace for $D_{8 t}, S_{8 t}$ and $M_{8 t}$

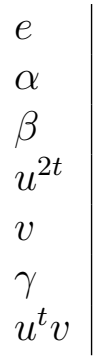

Table 2: Partial 2-sequencing for $D_{8 t}$

$$
\begin{array}{r}
u^{2 t-1}, u v, u^{2 t-2}, u^{2} v, u^{2 t-3}, \ldots, u^{t+1}, u^{t-1} v, u^{t} \\
u^{t+1} v, u^{t-1}, u^{t+2} v, u^{t-2}, \ldots, u^{2}, u^{2 t-1} v, u, u^{2 t} v \\
u^{2 t} \\
v \\
u^{2 t+1} v, u^{4 t-1} v, u^{2 t+2} v, u^{4 t-2} v, \ldots, u^{3 t-1} v, u^{3 t+1} v, u^{3 t} v \\
u^{t} v
\end{array}
$$

The associated partial 2-sequencings arising from the partial terrace for $D_{8 t}, S_{8 t}$ and $M_{8 t}$ are given in Tables 2, 3 and 4 respectively with each row starting with the difference created by joining the subsequence with the previous one.

In each case, to complete the sequence to a terrace $\delta$ needs to satisfy three conditions. First, it must generate the final quotient of the form $u^{x} v$, which it can do by starting with $u^{3 t-1}$. Second, it must contain the elements of the form $u^{x}$ for $2 t+1 \leqslant x \leqslant 4 t-1$. Third, it must generate one from each inverse pair within $\langle u\rangle$ except for $u^{2 t}$ and $u^{ \pm(2 t-1)}$. Further, for the terrace to be extendable, the last element of $\delta$ must be $u^{4 t-2}$.

These conditions can be met by taking a graceful sequence $\left(g_{1}, g_{2}, \ldots, g_{2 t-1}\right)$ that starts with $t-2$ and ends with $2 t-3$ and defining the $i$ th element of $\delta$ to be $u^{2 t+1+g_{i}}$. Such a sequence exists by Lemma 2 .

Finally, we need to check the other condition to be extendable; that $a_{j-1} a_{j+1}=a_{j}=$ $a_{j+1} a_{j-1}$ for some $j \geqslant 5$. Setting $j=4 t-1$, we find that $a_{j-1}=u^{2 t} v, a_{j}=u^{2 t}$ and $a_{j+1}=v$; a valid choice in each of the three groups.

Example 3 The terrace for $D_{32}, S_{32}$ and $M_{32}$ given by Theorem 3 is $e, u^{7}, u v, u^{6}, u^{2} v, u^{5}, u^{3} v, u^{4}, u^{5} v, u^{3}, u^{6} v, u^{2}, u^{7} v, u, u^{8} v, u^{8}$

$$
v, u^{9} v, u^{15} v, u^{10} v, u^{14} v, u^{11} v, u^{13} v, u^{12} v, u^{4} v, u^{11}, u^{13}, u^{12}, u^{9}, u^{15}, u^{10}, u^{14} .
$$

When considering which groups are most likely to give a counterexample to Bailey's conjecture those with many involutions and/or large elementary abelian 2-groups as subgroups 
Table 3: Partial 2-sequencing for $S_{8 t}$

$$
\begin{aligned}
& \alpha \\
& \beta \\
& u^{2 t} \\
& v \\
& \gamma \\
& u^{t} v
\end{aligned}
$$

Table 4: Partial 2-sequencing for $M_{8 t}$

$$
\begin{aligned}
& \alpha \\
& \beta \\
& u^{2 t} \\
& v \\
& \gamma \\
& u^{t} v
\end{aligned}
$$

$$
\begin{array}{r}
u^{2 t-1}, u^{2 t+2} v, u^{3} v, u^{2 t+4} v, u^{5} v, \ldots, u^{4 t-2} v, u^{2 t-1} v \\
u v, u^{2} v, u^{3} v, \ldots, u^{2 t-2} v, u^{2 t-1} v \\
v \\
u^{2 t} v \\
u^{4 t-1}, u^{2 t+2}, u^{4 t-3}, u^{2 t+4}, \ldots, u^{2 t+3}, u^{4 t-2}, u \\
u^{2 t}
\end{array}
$$

$$
\begin{array}{r}
u^{2 t-1}, u^{2 t+2} v, u^{4 t-3} v, u^{2 t+4} v, u^{4 t-5} v, \ldots, u^{4 t-2} v, u^{2 t+1} v \\
u v, u^{4 t-2} v, u^{3} v, u^{4 t-4}, \ldots, u^{2 t+2} v, u^{2 t-1} v \\
v \\
u^{2 t} v \\
u, u^{2 t-2}, u^{3}, u^{2 t-4}, \ldots, u^{2 t-3}, u^{2}, u^{2 t-1} \\
u^{2 t}
\end{array}
$$

are natural contenders. Theorem 3 and Corollary 1 imply that many such contenders are indeed terraced; groups of the form $\mathbb{Z}_{2}^{2 s} \times D_{8 t}$ for all $s$ and for $t \geqslant 2$, for example.

A computer search for extendable terraces for small groups has been implemented in GAP [5]. Neither of the two non-abelian groups of order 8 has an extendable terrace. Extendable terraces were found for all twelve non-abelian groups of orders 12, 16 and 20 not covered by Theorem 3. The notation $G_{n / p}$ indicates that the group has order $n$ and is in position $p$ in GAP's small group library. Where the group has a common name that is indicated as well, and we use the more familiar permutation notation for the alternating group $A_{4}$. The value for $j$ in the definition of an extendable terrace is also given.

Order 12:

$$
\begin{aligned}
G_{12 / 1}= & \left\langle a, b: a^{6}=e, b^{2}=a^{3}, a b=b a^{-1}\right\rangle \cong Q_{12}, j=5 \\
& e, a^{2}, a, b, a^{3} b, a^{3}, a^{4} b, a^{5}, a^{5} b, a b, a^{2} b, a^{4} \\
G_{12 / 3} \cong & A_{4}, j=7 \\
(),(123),(234),(124),(134),(14)(23),(12)(34),(13)(24),(142),(143),(2 & \\
G_{12 / 4}= & \left\langle a, b: a^{6}=b^{2}=e, a b=b a^{-1}\right\rangle \cong D_{12}, j=6 \\
& e, a, a^{2} b, a^{5}, a^{4} b, a b, a^{3}, a^{3} b, a^{5} b, b, a^{4}, a^{2}
\end{aligned}
$$


Order 16:

$$
\begin{aligned}
G_{16 / 3}= & \left\langle a, b, c: a^{4}=b^{2}=c^{2}=e, a b=b a c,[a, c]=[b, c]=e\right\rangle, j=6 \\
& e, a, a^{3}, a^{3} c, a^{2} b, b c, a^{2} c, b, a b, a c, a b c, c, a^{3} b c, a^{2} b c, a^{3} b, a^{2} \\
G_{16 / 4}= & \left\langle a, b: a^{4}=b^{4}=e, a b=b a^{-1}\right\rangle, j=13 \\
& e, a^{2} b, a^{3} b^{3}, a^{3}, a b^{3}, a^{2}, a, a^{3} b^{2}, b, b^{3}, a^{2} b^{3}, a^{3} b, a^{2} b^{2}, a b, a b^{2}, b^{2} \\
G_{16 / 9}= & \left\langle a, b: a^{8}=e, b^{2}=a^{4}, a b=b a^{-1}\right\rangle \cong Q_{16}, j=5 \\
& e, a^{2} b, a b, a, a^{7}, a^{6}, b, a^{3} b, a^{7} b, a^{5} b, a^{2}, a^{5}, a^{6} b, a^{3}, a^{4} b, a^{4} \\
G_{16 / 11}= & \left\langle a, b, c: a^{4}=b^{2}=c^{2}=e, a b=b a^{-1},[a, c]=[b, c]=e\right\rangle \cong D_{8} \times \mathbb{Z}_{2}, j=8 \\
& e, a^{3} c, a^{3}, a, b, a^{3} b, b c, a^{2} c, a^{2} b, c, a b, a^{3} b c, a^{2} b c, a c, a b c, a^{2} \\
G_{16 / 12}= & \left\langle a, b, c: a^{4}=c^{2}=e, b^{2}=a^{2}, a b=b a^{-1},[a, c]=[b, c]=e\right\rangle \cong Q_{8} \times \mathbb{Z}_{2}, j=10 \\
& e, b, a b, a c, b c, c, a, a^{3} c, a^{3} b, a^{2} c, a^{3} b c, a b c, a^{2} b, a^{2} b c, a^{3}, a^{2} \\
G_{16 / 13}= & \left.\left\langle a, b, c: a^{2}=b^{2}=c^{4}=e, a b=b a c^{2},[a, c]=[b, c]=e\right)\right\rangle, j=8 \\
& e, c, a c^{2}, a b c^{2}, b c^{3}, a c, b c, b, c^{3}, a b, b c^{2}, a c^{3}, a b c, a b c^{3}, a, c^{2}
\end{aligned}
$$

Order 20:

$$
\begin{aligned}
G_{20 / 1}= & \left\langle a, b: a^{10}=e, b^{2}=a^{5}, a b=b a^{-1}\right\rangle \cong Q_{20}, j=9 \\
& e, b, a^{6} b, a b, a^{4} b, a, a^{3} b, a^{7}, a^{6}, a^{9}, a^{2} b, a^{2}, a^{4}, a^{8} b, a^{7} b, a^{8}, a^{5} b, a^{9} b, a^{3}, a^{5} \\
G_{20 / 3}= & \left\langle a, b: a^{5}=b^{4}=e, a b=b a^{2}\right\rangle, j=17 \\
& e, a^{2}, a b, a^{3}, a^{4} b^{3}, a b^{3}, a^{2} b^{2}, a^{2} b, b^{2}, a, a^{4} b^{2}, b, a^{3} b, a^{3} b^{3}, a^{3} b^{2}, a b^{2}, a^{2} b^{3}, a^{4} b, b^{3}, a^{4} \\
G_{20 / 4}= & \left\langle a, b: a^{10}=b^{2}=e, a b=b a^{-1}\right\rangle \cong D_{20}, j=5 \\
& e, a^{6}, a^{4} b, a^{5}, a^{8} b, a^{3} b, b, a^{4}, a^{5} b, a^{8}, a^{2} b, a^{9} b, a^{7} b, a^{6} b, a, a b, a^{9}, a^{7}, a^{3}, a^{2}
\end{aligned}
$$

The smallest order for which Bailey's conjecture is not settled is 64 . The abelian case for this order was proven in $[9,11]$. Of the 256 non-abelian groups of order 64 , only three were known to have terraces prior to this work [8]. The extendable terraces for groups of order 16 above imply that at least 25 further non-abelian groups of order 64 are terraced (this is the number of groups that have commuting elements in all pairs of commuting cosets of some central Klein 4-group to use in Theorem 2). Combining this with the known ones and the new terraces here for $S_{64}$ and $M_{64}$ gives a total of 30. There are 208 non-abelian groups of order 64 that have a central Klein 4-group with at least one pair of commuting cosets that contain a pair of commuting elements; many of these may fall to Theorem 2 if an appropriate extendable terrace for the quotient group of order 16 can be found. 


\section{References}

[1] B. A. Anderson and E. C. Ihrig. All groups of odd order have starter-translate 2sequencings. Australas. J. Combin. 6 (1992) 135-146.

[2] B. A. Anderson and E. C. Ihrig, Symmetric sequencings of non-solvable groups, Congr. Numer. 93 (1993) 73-82.

[3] R. A. Bailey, Quasi-complete Latin squares: construction and randomization, J. Royal Statist. Soc. Ser. B 46 (1984) 323-334.

[4] J. A. Gallian, A dynamic survey of graph labellings, Electron. J. Combin. DS6 (20012010), 246pp.

[5] GAP group. GAP - Groups, Algorithms, and Programming, Version 4, 1999.

[6] Y.-S. Hwang, D. B. Leep and A. R. Wadsworth, Galois groups of order $2 n$ that contain a cyclic subgroup of order n, Pacific J. Math. 212 (2003) 297-319.

[7] É. Lucas, Récréations Mathémathiques, Tôme II, Albert Blanchard, Paris, 1892 (reprinted 1975).

[8] M. A. Ollis, Sequenceable groups and related topics, Electron. J. Combin. DS10 (2002) $34 \mathrm{pp}$.

[9] M. A. Ollis, On terraces for abelian groups, Disc. Math. 305 (2005) 250-263.

[10] M. A. Ollis, A note on terraces for abelian groups, Australas. J. Combin. 52 (2012), 229-234.

[11] M. A. Ollis and D. T. Willmott, On twizzler, zigzag and graceful terraces, Australas. J. Combin. 51 (2011) 243-257.

$[12]$ D. A. Preece, Zigzag and foxtrot terraces for $\mathbb{Z}_{n}$, Australas. J. Combin. 42 (2008) 261278.

[13] E. J. Williams, Experimental designs balanced for the estimation of residual effects of treatments, Aust. J. Scient. Res. A, 2 (1949) 149-168. 